\title{
Alternative food matrices for snack formulations in terms of acrylamide formation and mitigation
}

\author{
M. Mesías, C. Delgado-Andrade, F.J. Morales
}

Institute of Food Science, Technology and Nutrition, ICTAN-CSIC, Madrid 28040, Spain

\begin{abstract}
BACKGROUND: The salty snack market is re-inventing itself trying to offer to consumers healthier and added-value products often based on alternative vegetables/cereals, such as vegetable chips. New options mostly involve a nutritional improvement but the toxicological aspects of the reformulation are not usually examined. This work evaluated the acrylamide formation and effectiveness of different mitigation strategies in potato chips and in alternative snacks, such as carrot and pumpkin chips. Fresh potato, carrot and pumpkin samples were fried under controlled conditions and after being subjected to different operations (soaking in water at different times/temperatures, adding lemon or vinegar as $\mathrm{pH}$ modifiers or salt).

RESULTS: Acrylamide values in control samples of carrot (224 $\left.\pm 47 \mu \mathrm{gkg}^{-1}\right)$ and pumpkin $\left(5 \mathrm{l} 4 \pm 83 \mu \mathrm{gkg}^{-1}\right)$ were significantly lower than in the potato chip samples $\left(3887 \pm 509 \mu \mathrm{gkg}^{-1}\right)$. The different mitigation treatments had effects on potato and pumpkin chips, the most effective strategies being soaking options and the decrease of the $\mathrm{pH}$ of the medium. However, such treatments were not as effective in carrot chips.

CONCLUSIONS: These new vegetable snacks could be a healthy alternative both at nutritional and toxicological level. In addition, acrylamide concentration could even be reduced with simple mitigation operations before frying.
\end{abstract}

KEYWORDS: snack products; Maillard reaction; acrylamide; mitigation.

\section{INTRODUCTION}

Attending to the new preferences of an increasingly demanding consumer, salty snack companies are trying to redesign their products towards healthier and added-value alternatives. This has been the origin of the appearance in the market of new snacks based on novel pseudo-cereals, vegetables, roots or legumes that offer an improved nutritional profile and/or provide certain health-promoting benefit.'

Acrylamide is formed during thermal treatments applied in the snack industry, such as frying, extrusion or baking. ${ }^{2}$ This compound is produced as the result of the reaction between asparagine and reducing sugars as main precursors ${ }^{3}$ and has been identified as a public health concern due to its relation with different types of cancer. The European Food Safety Authority (EFSA) has worked for many years on the elaboration of different reports to make the food industry aware about the need of controlling the dietary acrylamide exposure. Since fried/roasted potatoes are the greatest exposition source to acrylamide in adulthood, ${ }^{3}$ a benchmark level of $750 \mu \mathrm{kgg}^{-1}$ of acrylamide for potato chips has been recently established, ${ }^{4}$ a value that could be reduced in the forthcoming years. Parallel, the new regulation underlines the importance of carrying out mitigation strategies along the whole production chain of potato transformation, including farmers, transport and storage of the fresh potato as well as pre-processing operations and processing conditions. ${ }^{4}$ Among the pre-processing operations, interventions are usually focused on lessen/inhibit the reaction between asparagine and reducing sugars by reducing the content of the reactants or modifying the conditions of the medium. In this sense, blanching at different temperatures or immersion of potatoes in solutions containing different acidifiers or salts with divalent cations are common procedures. ${ }^{5}$ 
Despite novel formulations of snacks offering an improved nutritional profile, it would be interesting to know the behavior of these new food matrices during thermal processing regarding the acrylamide formation. In addition, investigating the effectiveness of the mitigation strategies applied for potato products would help the snack industry to control acrylamide levels in the new formulations. The purpose of this brief study was to evaluate the acrylamide formation in fried carrot and pumpkin chips as new food matrices for healthy snack compared with fried potato. Different mitigation strategies were applied in the three vegetables with the aim of reducing the acrylamide exposure through novel vegetable snacks.

\section{MATERIALS AND METHODS}

\section{Reagents and chemicals}

A ${ }^{13} \mathrm{C}_{3}$-labelled acrylamide (99\% isotopic purity) was obtained from Cambridge Isotope Laboratories (Andover, MA, USA). Reversed-phase Oasis-HLB cartridges $(30 \mathrm{mg}, \mathrm{I} \mathrm{mL}$ )were obtained from Waters (Milford, MA, USA). All other chemicals, solvents and reagents were of analytical grade.

\section{Samples}

All the vegetables were acquired in supermarkets from Madrid (Spain): calibrated stored potato (50-80 $\mathrm{mm}$ ), Monalisa variety, origin France; calibrated carrot (I50-180 mm), category I, origin Spain; calibrated peanut pumpkin (240-300 mm), category I, origin Spain.

\section{Samples processing}

The three vegetables were peeled and cut into portions of $23 \mathrm{~mm}$ in diameter and $2 \mathrm{~mm}$ in thickness. The total slices were divided into nine groups of 65 slides which were submitted to different treatments as follows: Assay I (control): samples were not subjected to any mitigation strategy; Assay 2 (washing): samples were lightly washed; Assay 3 (cool soaking $10 \mathrm{~min}$ ): samples were soaked in $500 \mathrm{~mL}$ of room temperature water for $10 \mathrm{~min}$; Assay 4 (cool soaking $20 \mathrm{~min}$ ): samples were soaked in $500 \mathrm{~mL}$ of room temperature water for $20 \mathrm{~min}$; Assay 5 (cool soaking $30 \mathrm{~min}$ ): samples were soaked in $500 \mathrm{~mL}$ of room temperature water for $30 \mathrm{~min}$; Assay 6 (hot soaking): samples were soaked in $500 \mathrm{~mL}$ of water at $60^{\circ} \mathrm{C}$ for $10 \mathrm{~min}$; Assay 7 (lemon soaking): samples were soaked in $500 \mathrm{~mL}$ of room temperature water added with $25 \mathrm{~mL}$ of lemon

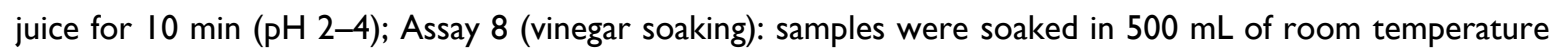
water added with $50 \mathrm{~mL}$ of vinegar for $10 \mathrm{~min}(\mathrm{pH} 3-4)$; Assay 9 (salt addition): I g of common salt was added to the 65 slices of vegetables. After different treatments, 50 slices of each assay were divided in two groups to perform the frying process in duplicate and the remaining slices were kept fresh, freeze-dried and properly stored until analysis. All the frying assays were performed with sunflower oil at $175^{\circ} \mathrm{C}$ for $2 \mathrm{~min}$ in a domestic fryer (Tefal, Frankfurt, Germany) of $3 \mathrm{~L}$ volume. Temperature was controlled with an external probe and frying time was established after a previous frying test (I, I.5, 2, 2.5 and $3 \mathrm{~min}$ ) to select the best organoleptic characteristics for the three type of vegetable chips. A similar temperature profile was observed for all the experiments.

\section{Moisture content of fresh and fried vegetables}

Moisture content of fried vegetables was determined in an oven-dryer at $105^{\circ} \mathrm{C}$ following the 950.46 Association of Official Analytical Chemists (AOAC) method.6 In the case of fresh samples, a close approximation to the moisture content was determined gravimetrically after freeze-drying instead of ovendrying, due to logistical limitations. Data were only used with the aim of establishing a correlation with the acrylamide content of samples.

\section{Determination of CIElab color in fried vegetables}


Measurements were made at room temperature using a HunterLab Spectrophotometer CM-3500D colorimeter (Hunter Associates, Stamford, CT, USA). Six independent measurements of a* (redness), b* (yellowness) and $L^{*}$ (lightness) parameters were obtained. Data of color were only used to establish correlations with the acrylamide content of samples.

\section{Determination of reducing sugars in fresh vegetables}

The determination of the reducing sugar (glucose + fructose) content was performed in freeze-dried samples as described in Mesías et al. ${ }^{7}$ Results were expressed as grams of glucose equivalents per kilogram of fresh sample. Analysis was done in duplicate.

\section{LC-ESI-MS/MS determination of acrylamide in fried vegetables.}

Sample extraction and clean up were based on the method described by Mesías and Morales. ${ }^{8}$ An Agilent 1200 liquid chromatograph coupled to an Agilent Triple Quadrupole MS detector (Agilent Technologies, Palo Alto, CA, USA) was used for the analyses. Further details on separation, conditions and quantification can be consulted at Mesías et al. ${ }^{9}$ Analysis was performed in duplicate and results were expressed as micrograms per kilogram of fried samples.

\section{Statistical analysis}

Statistical analyses were performed using SPSS version 23 (SPSS, Chicago, IL, USA). Analysis of variance (ANOVA) test was used to identify the overall significance of differences between different assays and vegetables. Differences were considered significant at $P<0.05$. Relationships between the different parameters analyzed were evaluated by computing Pearson correlation coefficients at the $P<0.05$ confidence level.

\section{RESULTS}

\section{Acrylamide content in the control samples of potato, carrot and pumpkin (Assay I)}

The acrylamide content in the control samples of the three fried vegetables is depicted in Fig. I (none mitigation strategy was applied). Fried potato showed significantly the highest levels of acrylamide (3887 \pm $\left.509 \mu \mathrm{kgg}^{-1}\right)$, followed by pumpkin $\left(5 \mathrm{I} 5 \pm 83 \mu \mathrm{gkg}^{-1}\right)$ and carrot $\left(225 \pm 48 \mu \mathrm{gkg}^{-1}\right)(\mathrm{P}<0.05)$. There is a lack of information concerning the formation of acrylamide in fried carrot and pumpkin as novel food matrices for salty snack formulation. Only the study by Takatsuki et al. ${ }^{10}$ described that potato matrix was more prone to acrylamide formation than pumpkin or carrot when they were baked at $220^{\circ} \mathrm{C}$ for $5 \mathrm{~min}$ (around 360, 230 and $<9 \mathrm{\mu gkg}^{-1}$ for potato, pumpkin and carrot, respectively). This decreasing order of acrylamide formation was well-supported by the asparagine content in the raw vegetables $(2.77,0.40$ and $0.10 \mathrm{~g} \mathrm{~kg}^{-1}$, respectively). Concerning the reducing sugar levels, all of them were statistically different in the fresh samples of the present study, having the pumpkin the highest values, followed by carrot and potato (Table I). Similar reducing sugar levels have been described previously in pumpkin," ${ }^{1}$ carrot $^{12}$ and in stored potatoes. ${ }^{7}$ Mesías et al..$^{7}$ established a correlation between acrylamide in fried potatoes and reducing sugar content in fresh potatoes. Attending to this correlation and taking into account the reducing sugar concentration found in the potato samples of the present study, a theoretical acrylamide concentration of about $3000 \mu \mathrm{gkg}^{-1}$ could be expected, which is close to the real level detected (3887 $\left.\pm 509 \mu \mathrm{gkg}^{-1}\right)$. If acrylamide data are examined in the framework of the new Regulation (UE) 2017/2158,4 the benchmark level marked for potato chips $\left(750 \mu \mathrm{gkg}^{-1}\right)$ was completely surpassed in the case of potato, with a value within the range purposed by Shamla and $\mathrm{Nisha}^{13}$ in their study on acrylamide concentration in Indian potato-based snack $\left(82-4245 \mu \mathrm{gkg}^{-1}\right)$. However, carrot and pumpkin showed significantly lower values and 
below this benchmark level, suggesting both matrices could be possible alternatives in the formulation of healthier snacks in toxicological terms.

\section{Effectiveness of mitigation strategies on acrylamide formation in fried vegetables}

A high acrylamide level in all the potato samples of the present study (treated or untreated) was observed. This fact was definitively marked by the great presence of reducing sugars in the stored potato used (19.2 \pm $0.6 \mathrm{mgg}^{-1}$ ) which could come from a sweetening phenomenon occurring during storage. ${ }^{14}$ The concentration measured was more than six-fold the recommended level by FDE (Food Drink Europe) ${ }^{14}$ in potato intended for frying $\left(3 \mathrm{mgg}^{-1}\right)$. A strong positive correlation was found between acrylamide and reducing sugar content $(P=0.0026 ; r=0.9582)$, in agreement with results described by Mesías et al. ${ }^{7}$

Many studies have reported different mitigation strategies to reduce the formation of acrylamide, all of them being detailed and recommended to be applied by the Regulation 2158/2017. ${ }^{4}$ Mitigation strategies evaluated in the present study were selected according to these recommendations, including the reduction of the sugar content in the raw foods by washing and soaking (Assays 2-6), the reduction of the level of $\mathrm{pH}$ by the addition of lemon and vinegar in order to limit the reaction (Assays 7 and 8 ) and the addition of salt as a factor that influences the formation of processing contaminants (Assay 9). Mitigation strategies were quite effective in potato (Fig. 2(A)). Washing reduced acrylamide in $22.5 \%$ whereas soaking in cool water showed more inhibitory results, increasing with soak duration $(29.2 \%, 52.4 \%$ and $53.3 \%$ inhibition for 10,20 and $30 \mathrm{~min})$. Acrylamide reduction in hot soaking treatment (53.8\%) was equivalent to the cool soaking $30 \mathrm{~min}$. Accordingly, the application of longer blanching times $\left(50^{\circ} \mathrm{C} / 70 \mathrm{~min}\right.$ and $\left.70^{\circ} \mathrm{C} / 40 \mathrm{~min}\right)$ has shown important reductions in acrylamide content, varying between $91 \%$ and $97 \% .{ }^{15}$ The addition of lemon to the soaking water also exerted a mitigation effect (38.6\%), deeper in the case of vinegar (59.6\%). The main treatment responsible for acrylamide reduction should be the decrease of the reducing sugar content after soaking, excepting for vinegar soaking (Table I). According to Pedreschi et al., ${ }^{15}$ the immersion of potato slices in citric acid did not affect their asparagine or reducing sugars content, but there was a significant reduction in the content of acrylamide (70\%). This decrease may be attributed to the $\mathrm{pH}$ diminution, showing a $99 \%$ of acrylamide mitigation for $\mathrm{pH} 4$ versus $\mathrm{pH} \mathrm{7.}{ }^{16}$ The presence of salt also induced a lessened acrylamide content, with a drop of $38.7 \%$ as previously established by Moreau et al. ${ }^{17}$ Significant correlations were found between acrylamide and $L^{*}(P=-0.0119 ; r=0.9097), a^{*}(P=0.0002 ; r=0.9890)$ and $b^{*}(P=0.0154 ; r=$ 0.8967), supporting the tight link between acrylamide formation with the color development in fried potatoes.

In carrots, none of the soaking treatments in cool water reduced the acrylamide content (Fig. 2(B)), it was even increased in some cases. It must be mentioned that the lower size of carrots compared with potato tubers led to the use of a high amount of pieces to collect the entire pool of carrot slices for the assays and the initial precursors content in those pieces, specially reducing sugars, could be different, as Table I depicts. In the hot soaking treatment, a slight decrease in the acrylamide level ( $\approx 8 \%$ reduction) consequent to a lower reducing sugar content in the treated fresh carrot was observed. This finding would be in line with results from Bao and Chang, ${ }^{18}$ who registered the same effect after blanching of carrots. Lemon and vinegar soaking led to a lower formation of acrylamide in the fried carrot ( $31.0 \%$ and $46.4 \%$ inhibition, respectively), which was accompanied by the decline in the reducing sugar content, especially significant for vinegar soaking $(P<0.05)$. Besides the acrylamide formation was markedly mitigated by the salt addition $(50.5 \%$ reduction), a parallel decrease in the reducing sugar content was not observed. 
Excepting washing and vinegar soaking treatments, an important decline in the acrylamide content was detected with mitigation strategies applied to pumpkin, ranging from $14.6 \%$ to $60.5 \%$ (Fig. 2(C)). Lemon soaking and salt addition were the most efficient treatments, although other strategies were also significantly good. Reducing sugars were homogeneously and significantly decreased in the treated fresh pumpkin $(\mathrm{P}<$ 0.05), however this parameter was not correlated with acrylamide in fried samples $(P=0.0943)$. In contrast, a significant and expected inverse relationship was observed between acrylamide concentration and the $L^{*}$ value $(P=0.0117 ; r=-0.9103)$.

\section{CONCLUSIONS}

This work evaluated the acrylamide formation and effectiveness of different mitigation strategies in potato chips and in alternative snacks, such as carrot and pumpkin chips. Since the presence of reducing sugars and asparagine (precursors) are combined with the application of high temperatures for frying, acrylamide formation took place in the three food matrices. The presence of the contaminant was significantly higher in potato chips compared with carrot and pumpkin chips, which exhibited levels below the benchmark level for potato chips established by the new acrylamide regulation. The mitigation operations applied were quite effective for potato and pumpkin samples, being different soaking options and the decrease of the $\mathrm{pH}$ of the medium the most effective strategies. However, such treatments were not as effective in carrot chips, although acrylamide level was in all cases far below the recommended level for potato chips. Therefore, these new snack matrices could be an alternative both at nutritional and toxicological level for the salty snack market. In addition, acrylamide concentration could even be reduced with simple mitigation strategies before frying.

\section{ACKNOWLEDGEMENTS}

This research was funded by the project CSIC-2017701025 (Spanish National Research Council) and SI013/ABI-3028-AVANSECAL (CAM). The authors thank Ms Laura Burguillo for the participation in the laboratory tasks and Ms I. Alvarez and Ms B. Díaz for their technical assistance.

\section{DECLARATION OF INTEREST STATEMENT}

The authors declare that they have no conflict of interest.

\section{REFERENCES}

I. Niva M, All foods affect health: understandings of functional foods and healthy eating among healthoriented Finns. Appetite 48:384-393 (2007).

2. Cheng WC, Sun DC, Chou SS and Yeh Al, Acrylamide content distribution and possible alternative ingredients for snack foods. J Food Prot 75:2158-2162 (20I2).

3. EFSA (European Food Safety Agency), Scientific opinion on acrylamide in food. EFSA J 13:4104 (2015) Available: http://www.efsa.europa.eu/sites/default/files/scientific_output/files/main_documents/4I04.pdf.

4. EC (European Commission), Commission Regulation (EU) 2017/2158 of 20 November 2017 Establishing Mitigation Measures and Benchmark Levels for the Reduction of the Presence of Acrylamide in Food. OJEU L304, pp. 24-44 (2017).

5. FDE (Food Drink Europe), Acrylamide Toolbox (2013). Available: http://fooddrinkeurope.eu/uploads/publications_documents/AcrylamideToolbox_20I3.pdf.

6. AOAC, Official Methods of Analysis of AOAC International, 16th edn. AOAC International, Washington, DC (1995). 
7. Mesías M, Holgado F, Márquez-Ruiz G and Morales FJ, Impact of the characteristics of fresh potatoes available in-retail on exposure to acrylamide: case study for French fries. Food Control 73:1407-1414 (2017).

8. Mesías M and Morales FJ, Acrylamide in commercial potato crisps from Spanish market: trends from 2004 to 2014 and assessment of the dietary exposure. Food Chem Toxicol 81:104-110 (2015).

9. Mesías M, Delgado-Andrade C, Holgado F and Morales FJ, Acrylamide content in French fries prepared in households: a pilot study in Spanish homes. Food Chem 260:44-52 (2018).

10. Takatsuki S, Nemoto S, Sasaki K and Maitani T, Production of acrylamide in agricultural products by cooking. Shokuhin Eiseigaku Zasshi 45:44-48 (2004).

11. Muzzaffar S, Baba WN, Nazir N, Masoodi FA, Bhat MM and Bazaz R, Effect of storage on physicochemical, microbial and antioxidant properties of pumpkin. Cogent Food Agric 2:I 163650 (2016).

12. McKee JMT, A simple method for the extraction of reducing and non-reducing sugars from carrot and other storage root vegetables. J Sci Food Agric 36:55-58 (1985).

13. Shamla $L$ and Nisha P, Acrylamide in deep-fried snacks of India. Food Addit Contam Part B-Surveill 7:220-225 (2014).

14. Sra SK, Sandhu KS and Ahluwalia P, Effect of treatments and packaging on the quality of dried carrot slices during storage. J Food Sci Technol 5I:645-654 (2014).

15. Pedreschi F, Kaack K and Granby K, Acrylamide content and color development in fried potato strips. Food Res Int 39:40-46 (2006).

16. Jung MY, Choi DS and Ju JW, A novel technique for limitation of acrylamide formation in fried and baked corn chips and in French fries. J Food Sci 68: 1287-1290 (2003).

17. Moreau L, Lagrange J, Bindzus W and Hill S, Influence of sodium chloride on colour, residual volatiles and acrylamide formation in model systems and breakfast cereals. Int J Food Sci Technol 44:2407-24l6 (2009).

18. Bao B and Chang KC, Carrot pulp chemical composition, color, and water holding capacity as affected by blanching. J Food Sci 59:I I59-I I6I (1994). 
FIGURES AND TABLES

Table I. Reducing sugar content in fresh samples (Control and after different treatments)

\begin{tabular}{lccc}
\hline \multirow{2}{*}{ Assay } & \multicolumn{3}{c}{ Reducing sugars (mg/g) } \\
\cline { 2 - 4 } & Potato & Carrot & Pumpkin \\
\hline I Control & $19.2 \pm 0.6 \mathrm{~cd}$ & $23.1 \pm 1.2 \mathrm{bc}$ & $31.2 \pm 0.3 \mathrm{e}$ \\
2 Washing & $18.7 \pm 0.2 \mathrm{c}$ & $20.6 \pm 0.7 \mathrm{ab}$ & $26.0 \pm 1.5 \mathrm{~cd}$ \\
3 Cool soaking $10 \mathrm{~min}$. & $17.8 \pm 0.5 \mathrm{~b}$ & $28.6 \pm 0.1 \mathrm{~d}$ & $22.3 \pm 0.1 \mathrm{~b}$ \\
4 Cool soaking $20 \mathrm{~min}$. & $17.3 \pm 0.1 \mathrm{ab}$ & $21.0 \pm 2.7 \mathrm{ab}$ & $18.9 \pm 0.5 \mathrm{a}$ \\
5 Cool soaking $30 \mathrm{~min}$ & $17.0 \pm 0.1 \mathrm{a}$ & $19.7 \pm 0.3 \mathrm{a}$ & $18.7 \pm 0.7 \mathrm{a}$ \\
6 Hot soaking & $16.7 \pm 0.3 \mathrm{a}$ & $19.6 \pm 0.4 \mathrm{a}$ & $19.1 \pm 0.1 \mathrm{a}$ \\
7 Lemon soaking & $18.0 \pm 0.3 \mathrm{~b}$ & $22.6 \pm 0.7 \mathrm{~b}$ & $24.4 \pm 0.4 \mathrm{c}$ \\
8 Vinegar soaking & $19.7 \pm 0.1 \mathrm{~d}$ & $18.9 \pm 0.8 \mathrm{a}$ & $25.1 \pm 0.4 \mathrm{~cd}$ \\
9 Salt addition & $24.8 \pm 0.1 \mathrm{e}$ & $25.4 \pm 0.8 \mathrm{c}$ & $26.7 \pm 1.8 \mathrm{~d}$ \\
\hline
\end{tabular}

Data are mean \pm SD. Different letters in the same column indicate significant differences $(p<0.05)$ 
Figure I. Acrylamide concentration in the fried potato, carrot and pumpkin (Assay I: Control). The benchmark value for potato chips according to the Regulation 2017/2158 is marked in dotted line. (750 $\mu g / \mathrm{kg})$. Different letters indicate significant differences $(p<0.05)$.

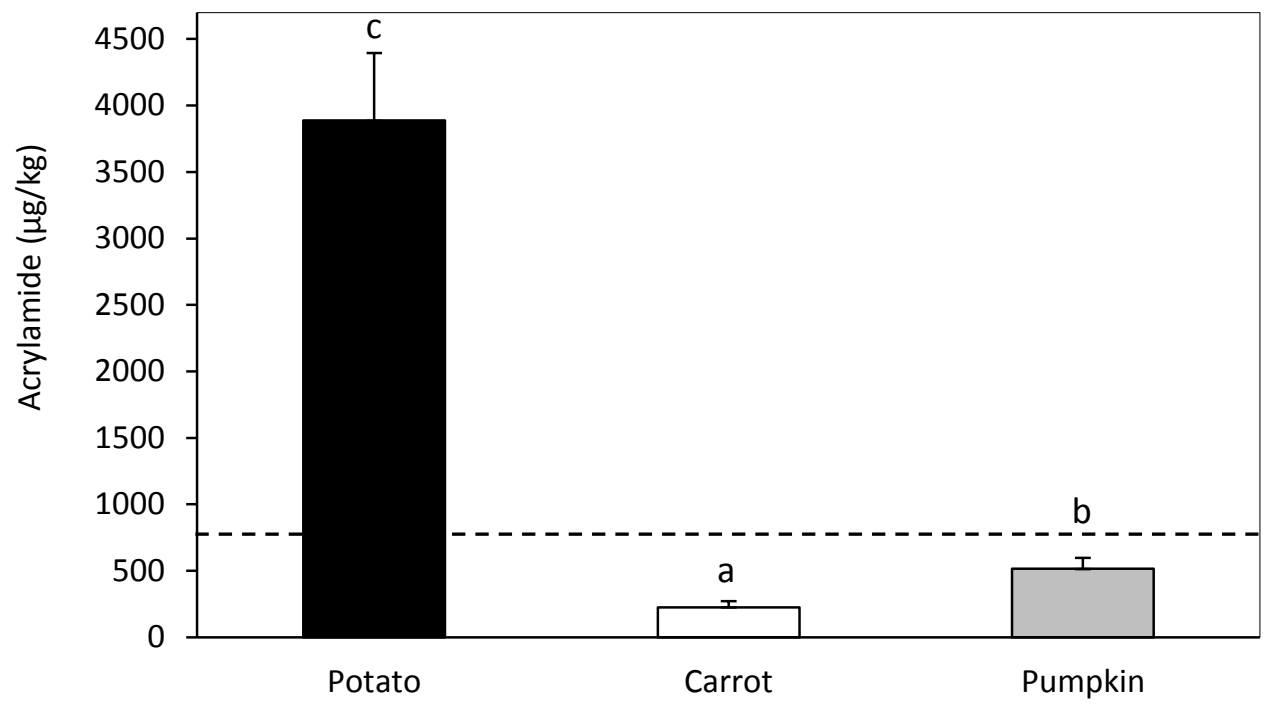


Figure 2. Acrylamide present (\%) in fried potato, carrot and pumpkin after applying diverse mitigation strategies (Control assay established as 100\% of acrylamide content). Different letters indicate significant differences $(p<0.05)$.
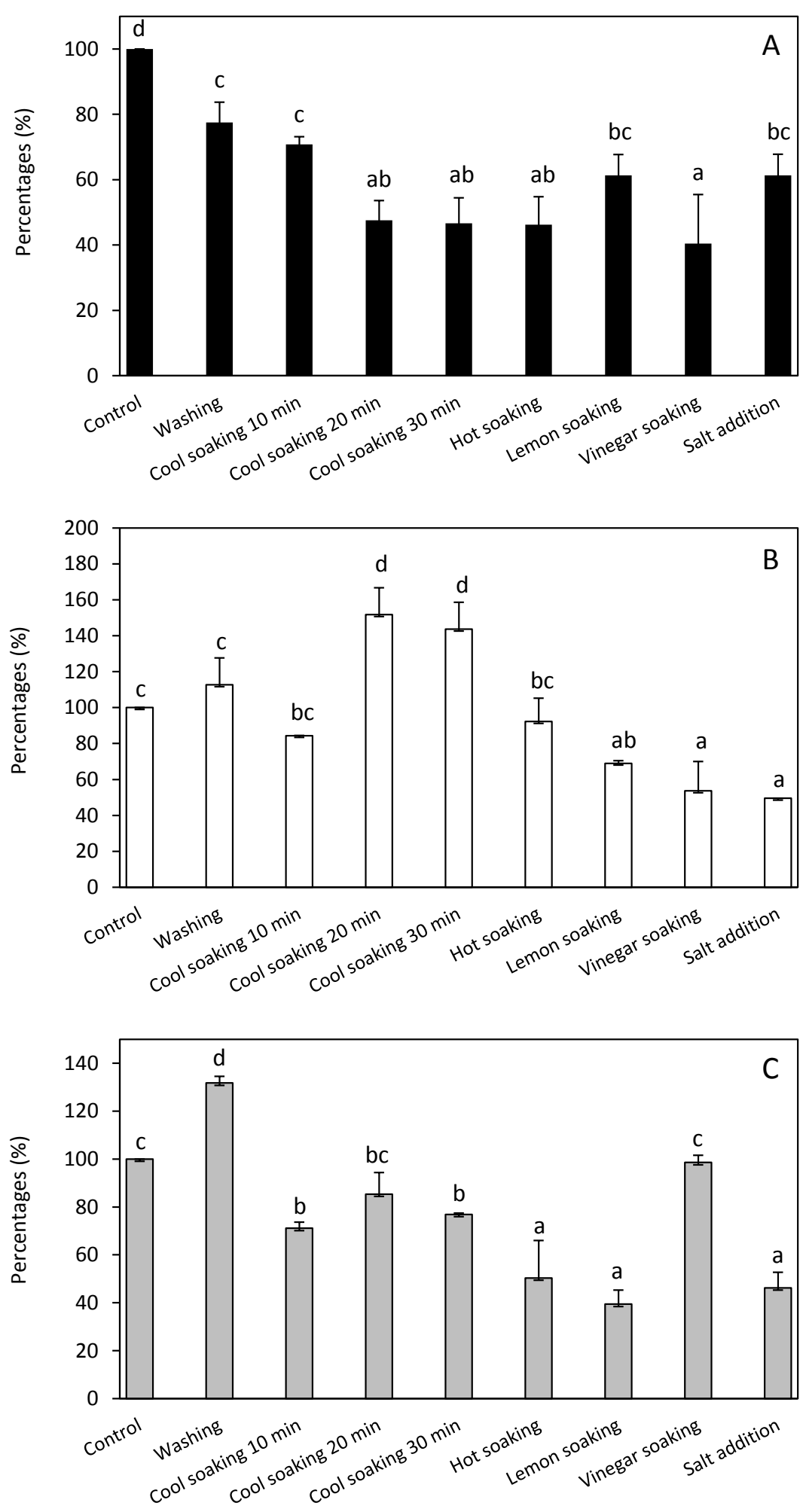